\title{
Sorafenib Inhibits Renal Fibrosis Induced by Unilateral Ureteral Obstruction via Inhibition of Macrophage Infiltration
}

\author{
Wenting Ma ${ }^{\mathrm{a}}$ Le Tao ${ }^{\mathrm{a}}$ Xuefei Wang ${ }^{\mathrm{b}}$ Qinyi Liu ${ }^{\mathrm{a}}$ Wei Zhang ${ }^{\mathrm{c}}$ Qiuying Li $^{\mathrm{d}}$ \\ Chunfeng He ${ }^{e}$ Dongying Xue ${ }^{a}$ Jie Zhanga Cheng Liuc \\ ${ }^{a}$ Department of Infectious Disease, ${ }^{b}$ Department of Digestive Disease, 'Laboratory of Molecular \\ Pathology, Central Laboratory, dDepartment of General Surgery, eDepartment of Urology, Putuo \\ Hospital, Shanghai University of Traditional Chinese Medicine, Shanghai, China
}

\section{Key Words}

Renal fibrosis • Macrophage $\cdot$ Sorafenib

\begin{abstract}
Aims: Sorafenib, which has been used extensively for the treatment of renal cell cancer and advanced hepatocellular carcinoma (HCC), has also been shown to have antifibrotic effects in liver fibrosis. However, the effects of sorafenib on renal fibrosis are unknown. Therefore, we investigated whether sorafenib inhibited renal fibrosis in a mouse model of unilateral ureteral obstruction (UUO) and further explored the potential mechanism. Methods: Mice underwent UUO followed by vehicle or sorafenib treatment. The expression of CD68, a macrophage marker, and the pro-inflammatory cytokines, MCP1 and CXCR3, were immunohistochemically analyzed. The involvement of macrophages in the formation of renal fibrosis was studied using confocal microscopy. Results: Renal histopathology improved in the UUO-sorafenib mice. Sorafenib notably suppressed TGF- $\beta 1$-mediated renal fibrogenic effects. The mRNA and protein expressions of CD68, MCP1, and CXCR3 in the obstructed kidney were significantly decreased by sorafenib. Immunohistochemistry showed that CD68 and CXCR3 had a similar distribution, whereas MCP1 was observed predominantly in the tubular epithelial cells. Double immunofluorescence demonstrated that CD68-positive macrophages could co-localize with CXCR3. It also revealed that CXCR3 interacted with CXCL11 in the UUO mouse kidneys. Widespread adhesion of macrophages to myofibroblasts was markedly inhibited in UUOsorafenib mouse kidneys. Conclusions: Taken together, the results indicated that sorafenib had protective effects against renal fibrosis; its mechanism of action was associated with inhibition of macrophage infiltration via the CXCR3/CXCL11 pathway. These data suggest the clinical potential of sorafenib for treatment of renal fibrosis and illustrate the immunological mechanisms underlying the protective effects of sorafenib.
\end{abstract}




\section{Cellular Physiology Cell Physiol Biochem 2016;39:1837-1849

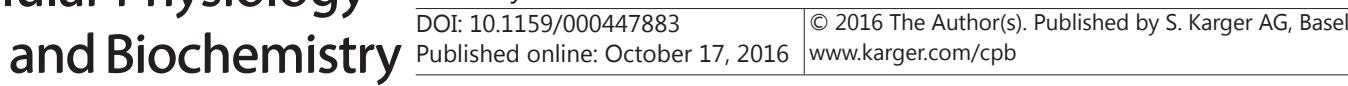 \\ Ma et al.: Sorafenib and Macrophage Infiltration}

\section{Introduction}

Renal fibrosis is a common feature of end-stage kidney disease, which occurs when a variety of renal diseases lead to renal failure [1]. The central cellular event during renal fibrosis progression is the accumulation of myofibroblasts, which are primarily responsible for the accumulation of extracellular matrix (ECM) in pathological conditions [2]. During renal fibrogenesis, macrophage infiltration has been shown to play important roles in the pathogenesis of renal fibrosis [3]. Macrophages produce a variety of pro-inflammatory cytokines, such as C-X-C chemokine receptor 3 (CXCR3) and monocyte chemoattractant protein 1 (MCP1), that promote the activation of myofibroblasts, which produce extracellular matrix proteins, especially collagen IV, and subsequently contribute to renal fibrosis [4]. Inhibiting macrophage infiltration may reduce the production of these inflammatory mediators; therefore, this may be a strategy to prevent progressive renal fibrosis leading to end-stage renal failure [5].

Sorafenib is a widely used multi-tyrosine kinase inhibitor that is approved by the FDA for treatment of hepatocellular carcinoma (HCC) and renal cell carcinoma [6]. Sorafenib induces apoptosis in various tumor cell lines and has been used to treat advanced HCC, cholangiocellular carcinoma and portal pressure [7-9]. Furthermore, sorafenib was shown to reduce portal hypertension in rats with experimentally induced fibrosis/cirrhosis [10, 11]. Sorafenib may be useful for the treatment of renal fibrosis through the suppression of TGF- $\beta /$ Smad-induced EMT signaling [12]. There is limited information about the effects of sorafenib on macrophage infiltration in renal fibrosis. Therefore, we investigated whether sorafenib reduced macrophage infiltration into the kidneys and renal fibrosis after unilateral ureteral obstruction (UUO) in mice.

\section{Materials and Methods}

\section{Reagents and antibodies}

Sorafenib was obtained from Alexis Biochemicals (San Diego, CA, USA). The Super Signal West Pico Chemiluminescent Substrate was from Thermo Scientific (Rockford, IL, USA), and the SYBR Green Supermix was from ABI (Glen Burnie, MD, USA). The SABC kit for immunohistochemical analysis was from Boster (Wuhan, China). Analytical grade chemicals and reagents were purchased from Sinopharmo Chemical and Reagents Co., Ltd (Shanghai, China) unless stated otherwise. A mouse monoclonal antibody to CD68 (MCA31R) was obtained from Serotec (Oxford, UK). A rabbit polyclonal antibody to CD68 (ab125212) was purchased from Abcam. A TNF- $\alpha$ rabbit antibody (ab1837p) was purchased from Millipore. MCP1 (sc1785), CXCR3 (sc-137140), CXCL11 (sc-28874), and p-Smad2 (sc-101801) were purchased from Santa Cruz Biotechnology (La Jolla, CA, USA). TGF- $\beta 1$ (ab66043), $\alpha$-SMA (ab5694), and Col IV (ab6586) were from Abcam (Pudong, Shanghai, China). Secondary fluorescence-labeled second antibodies were obtained from Jackson (West Grove, PA, USA). DAPI was from Sigma (Louis, MO, USA).

\section{Experimental protocol}

Thirty-two male C57BL/6J mice weighing 18 to $20 \mathrm{~g}$ were used in this study. Mice were maintained with free access to food and water under a constant $12 \mathrm{~h}$ light $/ 12 \mathrm{~h}$ dark photoperiod. The mice were divided into four groups (eight mice per group), including treated sham-operated (sham-vehicle), sorafenib-treated sham-operated (sham-sorafenib), treated UUO (UUO-vehicle) and sorafenib-treated UUO mice (UUOsorafenib). Renal fibrosis was induced in mice by UUO as previously described [13]. Briefly, under sodium pentobarbital anesthesia, the middle portion of the left ureter was ligated and cut between the two ligated points. When indicated, mice were treated with $5 \mathrm{mg} / \mathrm{kg}$ sorafenib daily by gavage. All procedures were approved by institutional review.

\section{Renal histology analysis}

Renal specimens were preserved in $4 \%$ paraformaldehyde and dehydrated in a graded alcohol series. Specimens were then embedded in paraffin blocks, cut into $3 \mu \mathrm{m}$-thick sections, and placed on glass slides. Sections were then stained with hematoxylin and eosin (HE) using a standard procedure, and tubule- 


\section{Cellular Physiology Cell Physiol Biochem 2016;39:1837-1849 \begin{tabular}{ll|l} 
and Biochemistry & $\begin{array}{l}\text { DOI: 10.1159/000447883 } \\
\text { Published online: October 17, } 2016\end{array}$ & $\begin{array}{l}\text { (c) 2016 The Author(s). Published by S. Karger AG, Basel } \\
\text { www.karger.com/cpb }\end{array}$ \\
\hline
\end{tabular} \\ Ma et al.: Sorafenib and Macrophage Infiltration}

interstitial injury was assessed according to the published criteria [14]. Masson and Sirius red were used for morphological observation of interstitial fibrosis. The Masson blue area indicating collagen and the red area of Sirius red staining, which represent the extent of the lesion, were calculated using Image Pro Plus 6.0.

\section{Hydroxyproline determination}

Renal hydroxyproline (Hyp) content was used as an indirect measure of tissue collagen content. Renal Hyp content was measured using a commercial detection kit (Jiancheng Institute of Biotechnology, Nanjing, China) according to the manufacturer's instructions. Hyp content was expressed as $\mu \mathrm{g} / \mathrm{gram}$ of wet kidney weight.

\section{Immunohistochemistry}

After deparaffinization and dehydration, microwave antigen retrieval was performed for 5 min before peroxidase quenching with $3 \% \mathrm{H}_{2} \mathrm{O}_{2}$ in phosphate-buffered saline (PBS) for $15 \mathrm{~min}$. Subsequently, the sections were blocked with 5\% BSA for $30 \mathrm{~min}$ and then incubated with each primary antibody overnight at $4^{\circ} \mathrm{C}$. After a wash in PBS, the sections were then incubated with biotinylated secondary antibodies for $30 \mathrm{~min}$ and then stained with 3, 3'-diaminobenzidine (DAB) for 2-5 min. Slides were counterstained with hematoxylin for 2-3 min, mounted, and examined.

\section{Laser confocal microscopy}

Immunofluorescence staining was performed on paraffin sections using different combinations of antibodies. For example, in the labeling of CD68, $\alpha$-SMA, and DAPI, kidney sections were first treated with monoclonal anti-CD68 for $1 \mathrm{~h}$ at room temperature. Slides were then washed three times with PBS and incubated with the secondary Cy3-conjugated Affinipure goat anti-mouse antibody for $30 \mathrm{~min}$. After washing, the sections were incubated with the $\alpha$-SMA antibody followed by FITC-conjugated Affinipure goat anti-rabbit antibody and then covered with mounting medium. Imaging analyses were performed using a Zeiss (Mannheim, Germany) LSM800 inverted stand and Axio Imager Z2m confocal system.

\section{Western blotting analysis}

Renal tissues were collected and frozen at $-80^{\circ} \mathrm{C}$ for western blot analysis. Briefly, the total proteins were separated by SDS-PAGE and transferred to a nitrocellulose membrane. The membrane was incubated overnight with primary antibodies against CD68, TNF- $\alpha$, MCP1, CXCR3 and GAPDH at $4{ }^{\circ} \mathrm{C}$, followed by incubation with HRP-conjugated secondary antibodies for $1 \mathrm{~h}$. Finally, the membrane was visualized with an enhanced chemiluminescence (ECL) advanced system (GE Healthcare, UK).

\section{Real-time PCR analysis}

Total RNA was extracted using TRIzol (Life Technologies, Grand Island, NY), followed by reverse transcription of total RNA to cDNA. cDNA was synthesized using a high-capacity cDNA reverse transcription kit (Applied Biosystems, Foster City, CA). cDNA subsequently underwent quantitative real-time polymerase chain reaction (PCR) using the ABI ViiA 7 Dx real-time PCR system (Life Technologies, Grand Island, NY). PCR primer sequences used were as follows: 18s rRNA forward 5'-AGTCCCTGCCCTTTGTACACA3', 18s rRNA reverse 5'-CGATCCGAGGGCCTCACTA-3'. CXCR3 forward 5'- TCTCGTTTTCCCCATAATCG3', CXCR3 reverse 5'- AGCCAAGCCATGTACCTTGA-3'. TNF- $\alpha$ forward 5'- AGGGTCTGGGCCATAGAACT-3', TNF- $\alpha$ reverse 5'- CCACCACGCTCTTCTGTCTAC-3'. MCP1 forward 5'- ATTGGGATCATCTTGCTGGT-3', MCP1 reverse 5'- CCTGCTGTTCACAGTTGCC-3'. CD68 forward 5'- ACCGCCATGTAGTCCAGGTA-3', CD68 reverse 5'- ATCCCCACCTGTCTCTCTCA-3'. TGF- $\beta 1$ forward 5'- AAGTTGGCATGGTAGCCCTT-3', TGF- $\beta 1$ reverse 5'GCCCTGGATACCAACTATTGC-3', Col I $(\alpha 1)$ forward 5'-TAGGCCATTGTGTATGCAGC-3', Col I $(\alpha 1)$ reverse 5'-ACATGTTCAGCTTTGTGGACC-3'. $\alpha$-SMA forward 5'-GTTCAGTGGTGCCTCTGTCA-3', $\alpha$-SMA reverse 5'ACTGGGACGACATGGAAAAG-3'. Col III $(\alpha 1)$ forward 5'- TGGTTCTGGCTTCCAGACAT-3', Col III $(\alpha 1)$ reverse 5'- CACCCTTCTTCATCCCACTC'-3', Col IV $(\alpha 1)$ forward 5'-CACGCCATGACAGTCACATT-3', Col IV ( $\alpha 1$ ) reverse 5'- GTCTGGCTTCTGCTGCTCTT-3'. Gene expression was normalized to 18s RNA as an internal control.

\section{Statistical analysis}

All results are expressed as the mean and standard deviation. Data were analyzed using a one-way analysis of variance (ANOVA, SPSS, Inc., Chicago, IL, USA). Groups were compared using ANOVA with Dunnett's multiple comparison test. Results with $\mathrm{p}<0.05$ were considered to be statistically significant. 


\section{Cellular Physiology Cell Physiol Biochem 2016;39:1837-1849

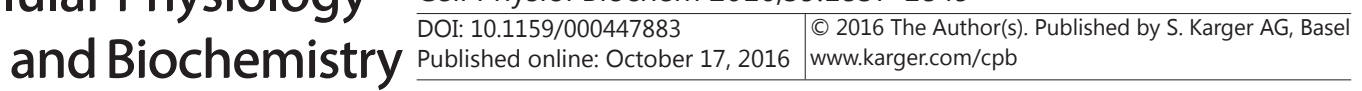 Ma et al.: Sorafenib and Macrophage Infiltration}

\section{Results}

\section{Histomorphological changes in obstructed kidneys}

To evaluate the possible antifibrotic or renoprotective effects of sorafenib in UUOinduced renal fibrosis, we administered sorafenib at the beginning of UUO. As shown in Figure $1 \mathrm{~A}$, the paraffin-embedded sections stained with H\&E exhibited significant tubulointerstitial damage, including tubular atrophy, inflammatory cell infiltration, and interstitial fibrosis, in UUO kidneys compared with those in sham kidneys. In contrast, tubulointerstitial damage and inflammatory response were reduced in UUO kidneys from sorafenib-treated mice compared with those of UUO-vehicle kidneys, as determined by semi-quantitative histomorphometry analysis (Fig. 1D).

UUO induced an increase in collagen as observed in the Masson's trichrome-stained kidney sections compared with sham-vehicle kidneys (Fig. 1B). However, sorafenib treatment in UUO kidneys resulted in lower collagen deposition compared with that of UUO-vehicle kidneys. This was also confirmed by semi-quantitative assessment of the blue area on the Masson's trichrome-stained kidney sections (Fig. 1E). The results of Sirius red staining showed that there was less collagen deposition around the renal tubules and that the fibrotic

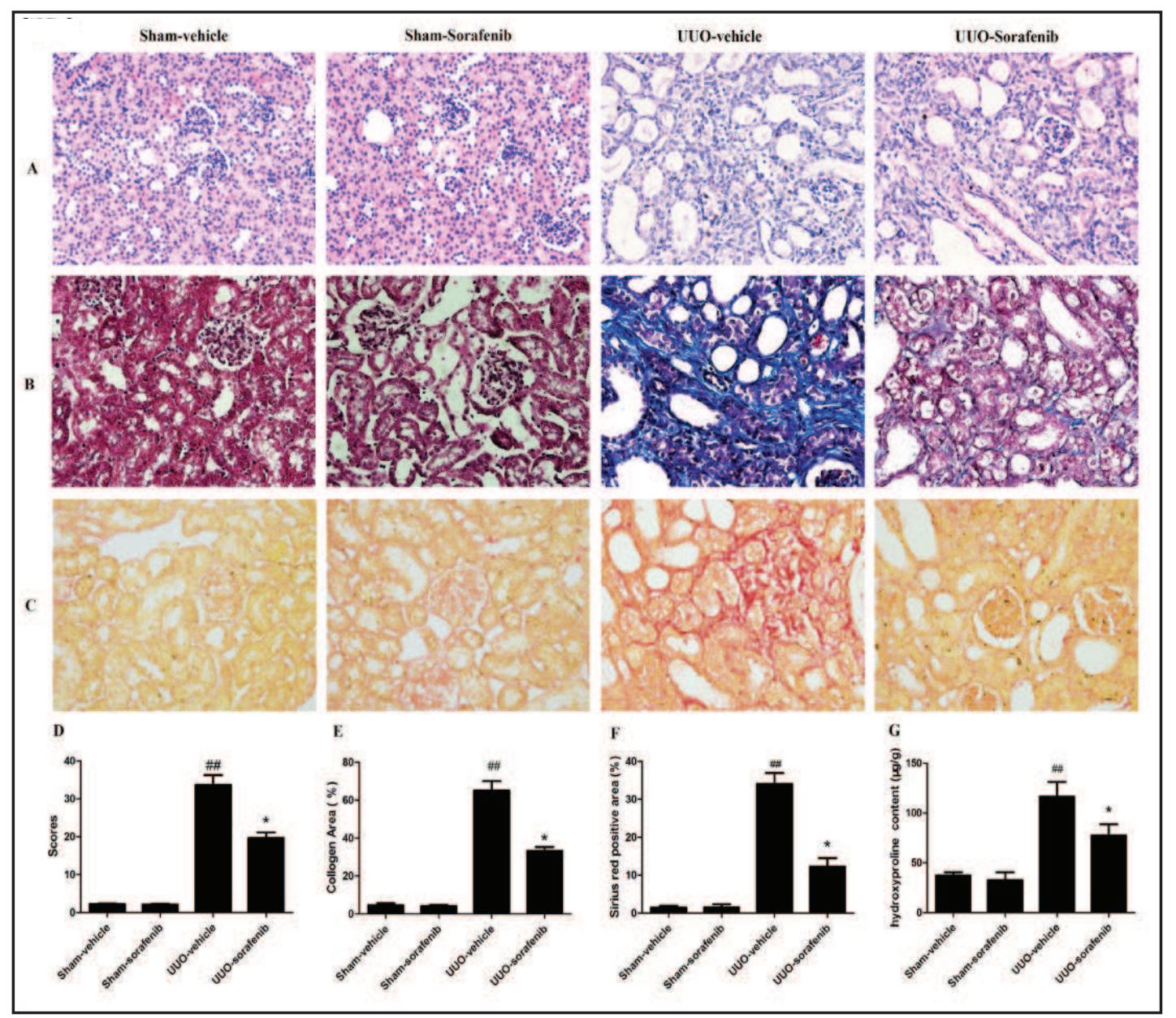

Fig. 1. Effects of sorafenib on renal histological changes in UUO-induced renal fibrosis. A, HE staining; B, Masson staining; C, Sirius red staining $(\times 400)$; D, histopathological scores within the tubulointerstitium from the sections; E, quantitative analysis of the extent of collagen (blue area \%) from the sections; F, quantitative analysis of Sirius red (red area \%) from the sections; G, Hyp content in renal tissue was measured. The data are shown as the mean \pm s.d. ${ }^{*} \mathrm{p}<0.05$, ${ }^{\# \#} \mathrm{p}<0.01$ vs sham-vehicle group; ${ }^{*} \mathrm{P}<0.05,{ }^{*} \mathrm{P}<0.01$ vs UUO-vehicle. 


\section{Cellular Physiology Cell Physiol Biochem 2016;39:1837-1849

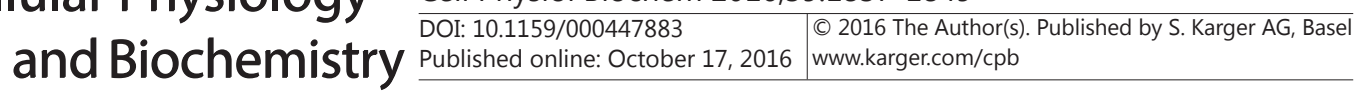

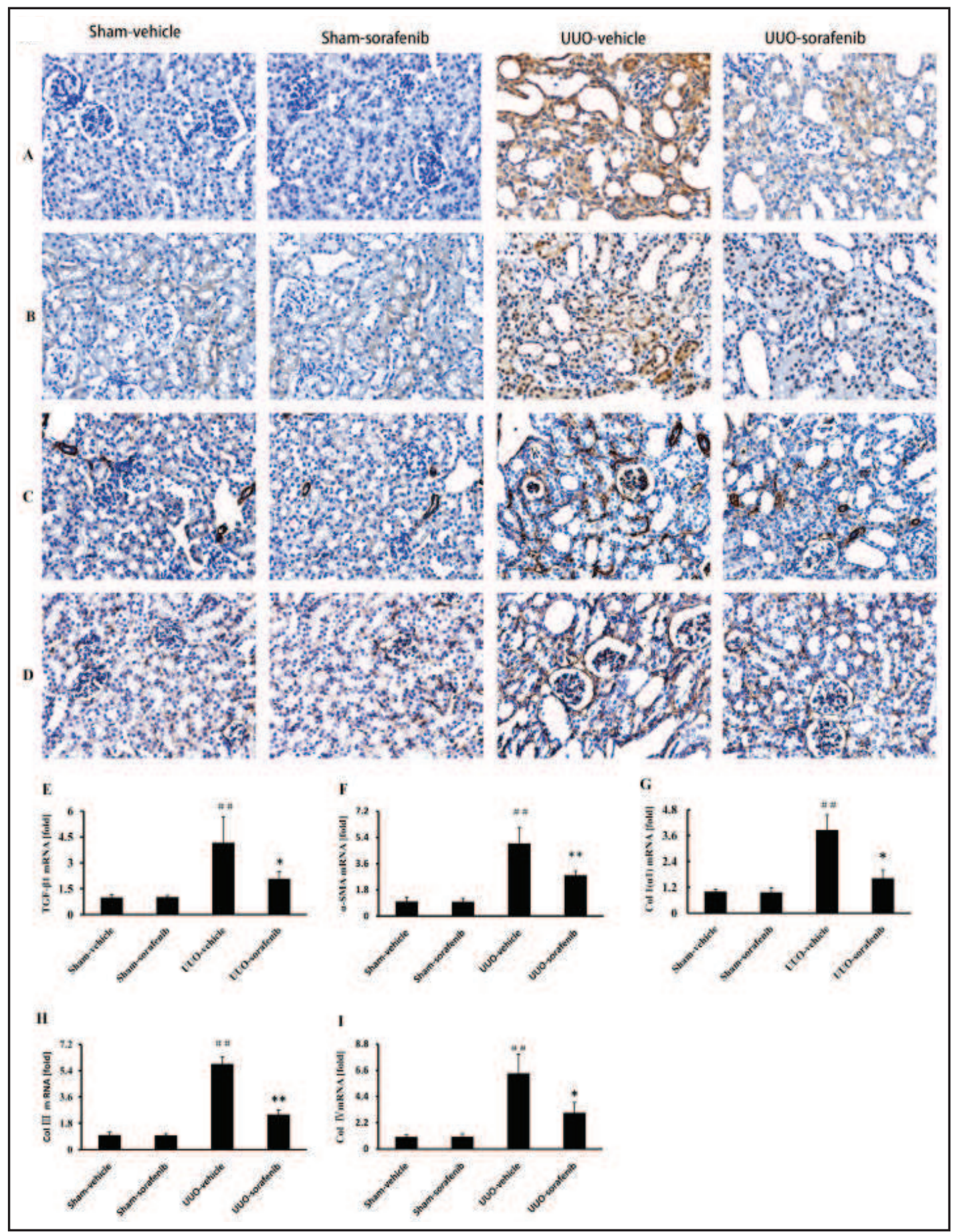

Fig. 2. Effects of sorafenib on pro-fibrogenic factors in the UUO mice model. A, TGF- $\beta 1$ staining; B, p-Smad2 staining; C, $\alpha$-SMA staining; D, Col IV staining; Brown staining indicates immunopositivity $(\times 400)$. (E-I) TGF- $\beta 1, \alpha$-SMA, Col I ( $\alpha 1$ ), Col III, and Col IV were measured by real-time PCR; as an internal control, 18S rRNA was amplified. The data are shown as the mean \pm s.d. ${ }^{\#} \mathrm{p}<0.05,{ }^{\#} \mathrm{p}<0.01$ vs sham-vehicle; ${ }^{*} \mathrm{p}<0.05$, ${ }^{* *} \mathrm{p}<0.01$ vs UUO-vehicle.

area in the UUO-sorafenib groups was significantly decreased compared with that in the UUO-vehicle group (Fig. 1C and 1F).

Changes in Hyp content in the kidney are considered an index of collagen metabolism and provide valuable information about the biochemistry and pathology of renal fibrosis. 


\section{Cellular Physiology Cell Physiol Biochem 2016;39:1837-1849 \begin{tabular}{ll|l} 
and Biochemistry & Published online: October 17, 2016 & $\begin{array}{l}\text { O } 2016 \text { The Author(s). Published by S. Karger AG, Basel } \\
\text { www.karger.com/cpb }\end{array}$ \\
\hline
\end{tabular}

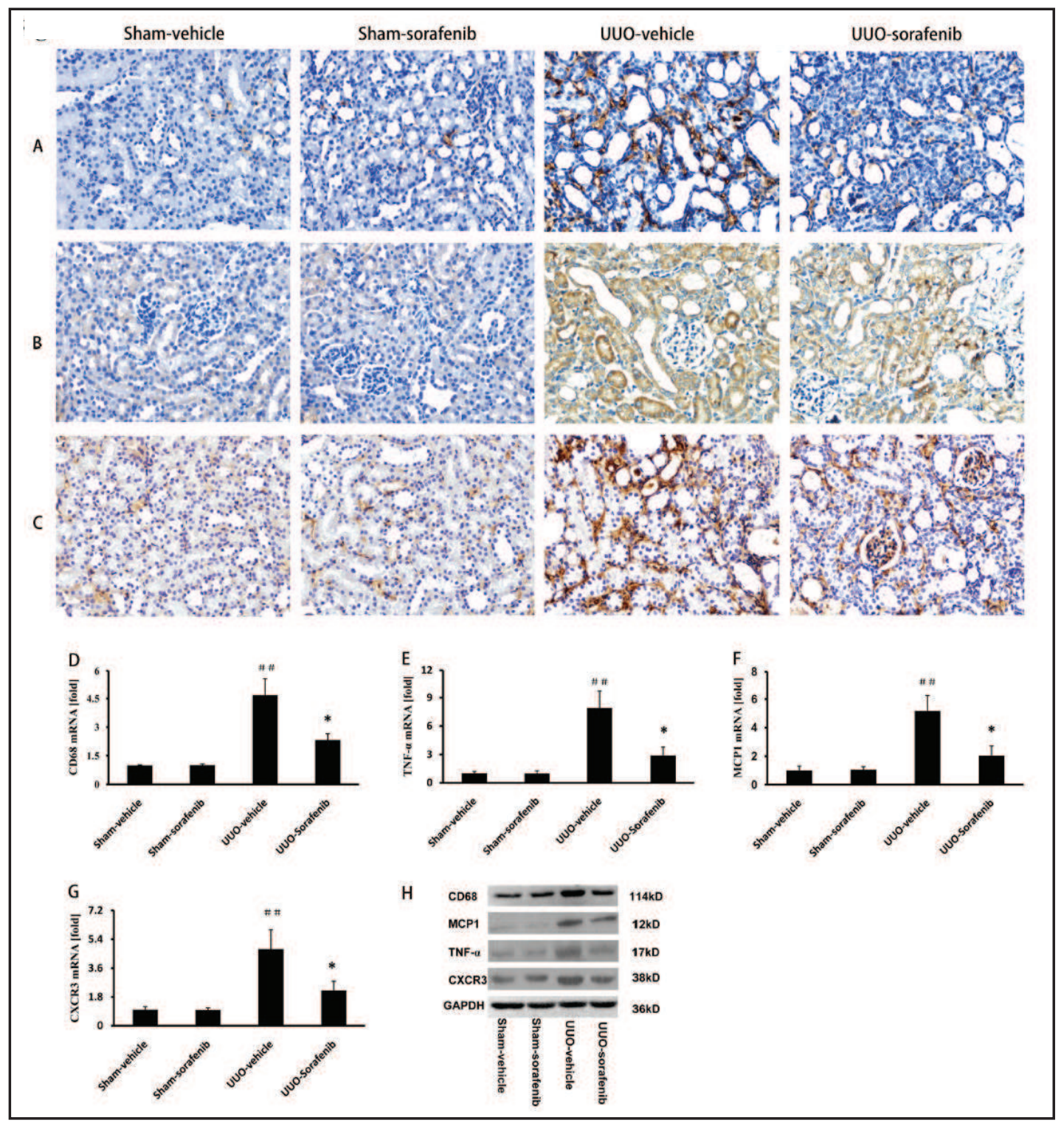

Fig. 3. Effect of sorafenib on macrophages and pro-inflammatory cytokines in the UUO mice model. A, CD68 staining; B, MCP1 staining; C, CXCR3 staining. Brown staining indicates immunopositivity $(\times 400)$. D-G, CD68, TNF- $\alpha$, MCP1, and CXCR3 detected by real-time PCR. As an internal control, 18S rRNA was amplified. H, CD68, TNF- $\alpha$, MCP1, and CXCR3 measured by western blot analysis. The data are shown as the mean \pm s.d. ${ }^{\#} \mathrm{p}<0.05,{ }^{\# \#} \mathrm{p}<0.01$ vs sham-vehicle; ${ }^{*} \mathrm{p}<0.05,{ }^{* *} \mathrm{p}<0.01$ vs UUO-vehicle.

As shown in Fig. 1G, the hydroxyproline content following 10 days of UUO treatment was approximately $311 \%$ of that of the sham-vehicle group ( $p<0.01$ ), suggesting abundant accumulation of collagen in the UUO-vehicle mice. In contrast, there was a 33\% reduction in hydroxyproline content $(\mathrm{p}<0.05)$ in the UUO-sorafenib group compared to the UUO-vehicle group, suggesting that sorafenib ameliorated renal collagen deposition in UUO-induced renal injury.

Effect of sorafenib on pro-fibrotic factors

As sustained deposition of extracellular matrix is predominantly due to the TGF- $\beta 1$ pathway and myofibroblast activation [15], TGF- $\beta 1 /$ pSmad $2 / \alpha$-SMA/Col IV were detected by immunohistochemical staining in the renal sections as described above. In sham mice, 


\section{Cellular Physiology Cell Physiol Biochem 2016;39:1837-1849 \begin{tabular}{ll|l} 
DOI: 10.1159/000447883 & @ 2016 The Author(s). Published by S. Karger AG, Basel
\end{tabular}}

and Biochemistry $\frac{\text { Published online: October 17, } 2016}{\mathrm{Ma} \text { et al.: Sorafenib and Macrophage Infiltratio }}$

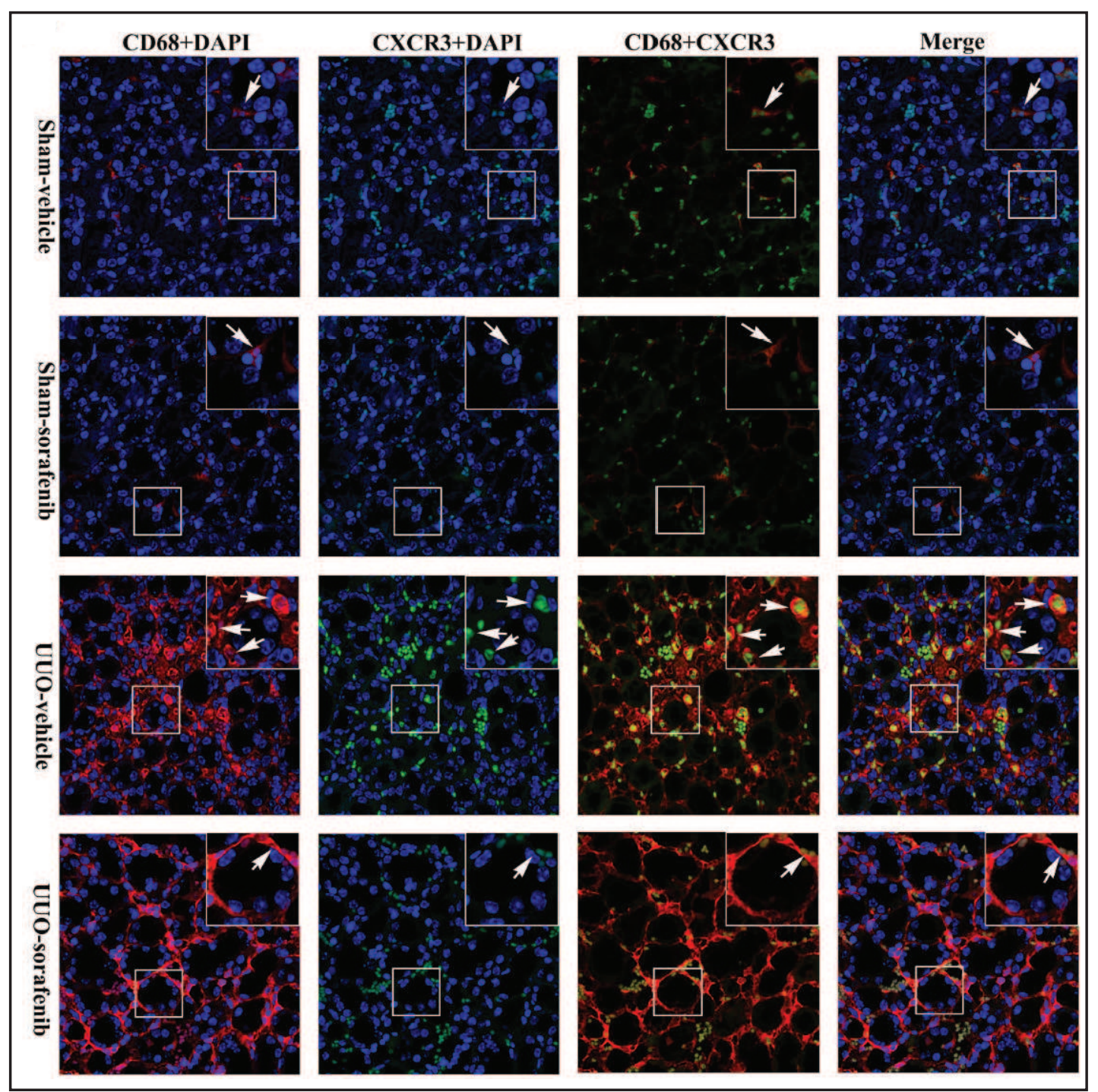

Fig. 4. Renal sections were stained for CD68 (red), CXCR3 (green), and DAPI (blue) and analyzed using laser confocal microscopy to determine the relationship between macrophages and CXCR3 expression in UUO-induced renal fibrosis. The arrow indicates CXCR3 derived from CD68-positive macrophages $(\times 400)$. The boxed area in the slide was enlarged and shown in the inset $(\times 800)$.

TGF- $\beta 1$ was very weakly stained and almost could not be detected, but TGF- $\beta 1$ increased significantly and was located mainly in the tubular epithelial, glomerular, and interstitial cells after 10 days of UUO treatment (Fig. 2A). Phosphorylation of Smad2 was weak and observed in the cytoplasm of the sham group, but p-Smad2 significantly increased, with strong staining in the nuclei of renal cells in UUO-vehicle mice, indicating that TGF- $\beta 1$ signaling was activated (Fig. 2B). The TGF- $\beta 1$ signaling pathway activates fibroblasts and increases ECM production. Thus, we detected $\alpha$-SMA and Col IV expression. As shown in Fig. 2C, only vascular smooth muscle cells were positive for $\alpha$-SMA in sham mice, and its expression markedly increased in pericytes, indicating their transition to myofibroblasts after UUO-treatment (Fig. 2C). Increased Col IV immunoreactivity in the kidneys of UUO mice was also decreased by sorafenib treatment (Fig. 2D). Similarly, renal expression of TGF- $\beta 1$, $\alpha$-SMA, and Col I, III, and IV mRNA was also demonstrated by real-time PCR analyses (Fig. 2E-I). 

Cellular Physiology Cell Physiol Biochem 2016;39:1837-1849 \begin{tabular}{c|l|l} 
DOI: 10.1159/000447883 & @ 2016 The Author(s). Published by S. Karger AG, Basel \\
and Biochemistry
\end{tabular} Ma et al.: Sorafenib and Macrophage Infiltration

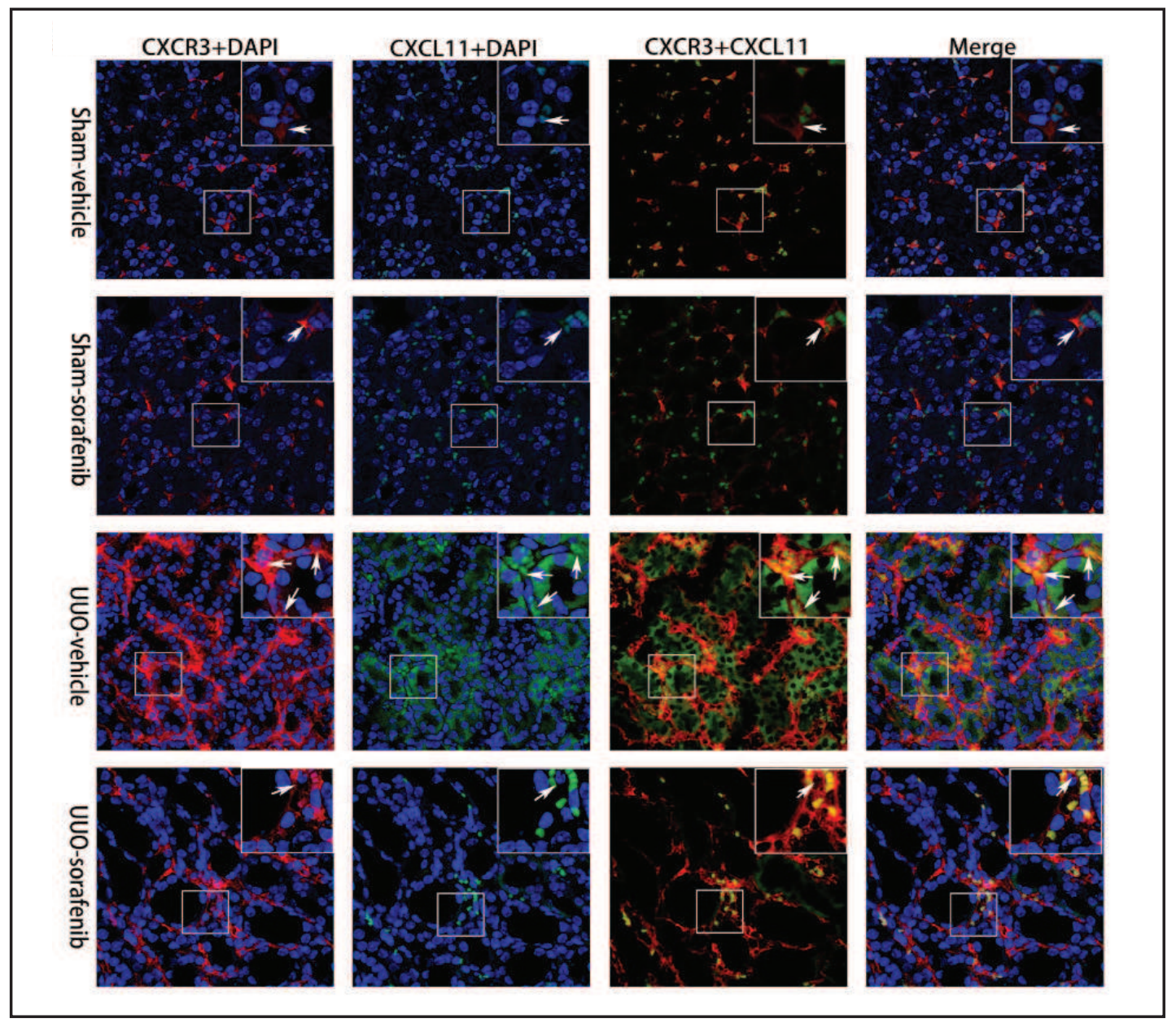

Fig. 5. Sorafenib inhibits the interaction between CXCR3 and CXCL11 in UUO-induced renal fibrosis in mice. Renal paraffin sections stained with CXCL11 (green), CXCR3 (red), and DAPI (blue); the arrow indicates CXCR3 and CXCL11 co-localization $(\times 400)$. The boxed area in the slide was enlarged and shown in the inset $(\times 800)$.

Taken together, these results confirm that UUO treatment induced TGF- $\beta 1$ pathway activation, myofibroblast activation, and accumulation of the extracellular matrix. Treatment with sorafenib in UUO ameliorated the obstructed kidneys and diminished the augmented expression of ECM-related factors.

Macrophage accumulation in renal fibrosis indicated by the specific markers CD68, MCP1 and CXCR3

Renal injury was associated with macrophage infiltration and triggered migration of macrophages into the cortical tubulointerstitium, where these macrophages stimulate fibrosis by interacting with myofibroblasts $[16,17]$. To elucidate the functions of macrophages in renal fibrosis, we used a specific macrophage marker, CD68, to monitor macrophage infiltration [18]. As shown in Fig. 3A, CD68-positive macrophage levels were low in the sham kidneys. CD68-positive macrophages with strong staining were located not only in glomerular but also in tubulointerstitium in UUO-vehicle mice.

As shown in Fig. 3B, MCP1 was predominantly located in the tubular epithelial cells with strong staining (Fig. 3B), indicating that MCP1 was not derived from macrophages in UUO-treated kidneys. The expression of CXCR3 was low in the sham kidneys (Fig. 3C), and CXCR3 appeared in the glomerular and cortical tubulointerstitium. Sorafenib treatment 

Cellular Physiology Cell Physiol Biochem 2016;39:1837-1849 \begin{tabular}{ll|l} 
DOI: 10.1159/000447883 & O 2016 The Author(s). Published by S. Karger AG, Basel \\
wwwkargercom/c(cp
\end{tabular} and Biochemistry Published online: October 17, 2016 www.karger.com/cpb

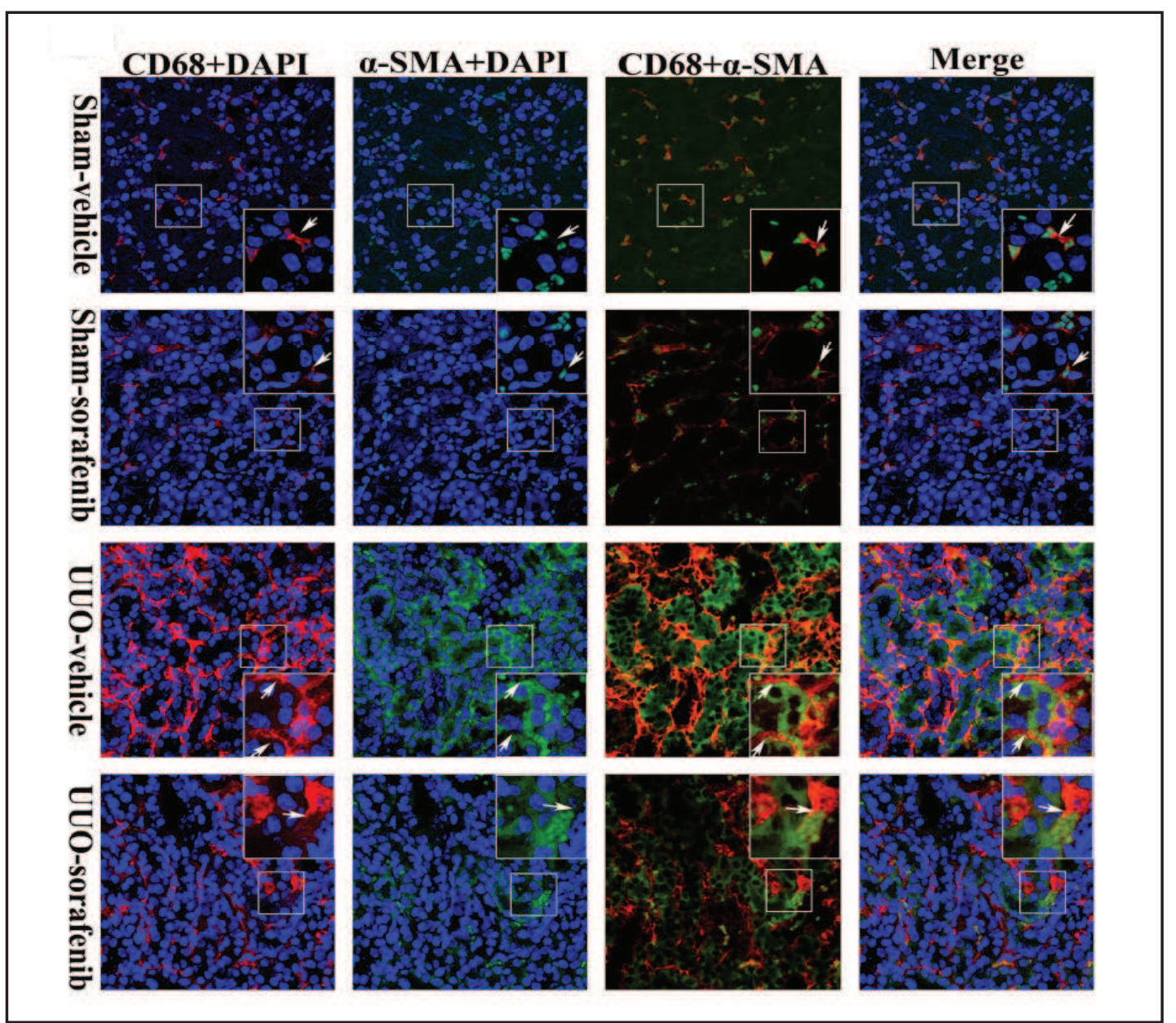

Fig. 6. Effect of sorafenib on macrophages and myofibroblast interaction. Renal paraffin sections stained with $\alpha$-SMA (green), CD68 (red), and DAPI (blue); the yellow color denotes co-localization $(\times 400)$. The boxed area in the slide was enlarged and is shown in the inset $(\times 800)$.

could suppress MCP1 and CXCR3 expression. The location of CXCR3 was very similar to that of CD68, suggesting that CXCR3 may be derived from CD68-positive macrophages.

Consistent with the results of immunostaining of CD68, MCP1, and CXCR3, increased expressions of CD68, TNF- $\alpha$, MCP1, and CXCR3 were further confirmed with real-time PCR and western blot analyses. Real-time PCR showed that CD68, TNF- $\alpha$, MCP1, and CXCR3 (Fig. 3D-G) transcripts were significantly up-regulated in UUO-vehicle mice. Sorafenib treatment markedly inhibited these pro-inflammatory cytokines compared with those of the UUOvehicle group $(p<0.05)$. Similar changes at the protein level of CD68 were observed in a western blot analysis (Fig. 3H).

These results suggest that macrophages infiltrated into the tubulointerstitium in renal fibrosis and are consistent with the hypothesis that macrophages are a major source of CXCR3, but not MCP1, production.

\section{Co-localization of CXCR3 with CD68 in UUO-induced renal fibrosis}

CXCR3 has an important function in the pathogenesis of liver fibrosis, and macrophages are the primary source of this cytokine [19]. However, there is little evidence indicating that CXCR3 was derived from macrophages in renal fibrosis in vivo. To this end, immunofluorescence analysis was performed. Double staining for CD68 and CXCR3 showed that CD68-positve macrophages produced CXCR3 in UUO-treated kidneys. As expected, 


\section{Cellular Physiology Cell Physiol Biochem 2016;39:1837-1849

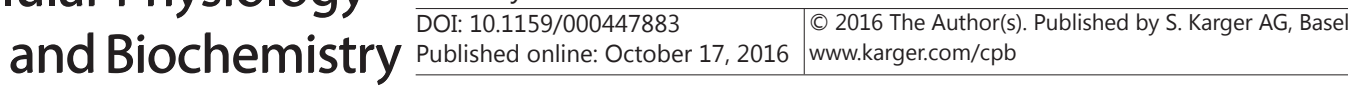 \\ Ma et al.: Sorafenib and Macrophage Infiltration}

there were a few double positive CD68 and CXCR3 cells in sham mice. Almost all CXCR3positive cells were found to be CD68-positive macrophages. In contrast, a lower number of infiltrating CD68-positive macrophages, which also had lower levels of CXCR3, was observed in the kidneys of UUO-sorafenib mice. These results showed that macrophages are the main producer of CXCR3 in UUO-induced renal fibrosis.

The CXCR3/CXCL11 axis mediates macrophage infiltration in UUO and is blocked by sorafenib

The number of macrophages was characteristically increased in the UUO-vehicle kidneys, and macrophages expressed CXCR3. Next, we determined which ligand (CXCL9, 10, and 11) combined with CXCR3 mediates macrophage infiltration. For this aim, we first examined the changes in these ligands by real-time PCR. The results showed that only CXCL11 increased significantly in UUO mouse kidneys (data not shown here) compared with that in sham mice. We hypothesized that CXCL11 may be involved in macrophage recruitment to the injured kidneys in UUO-treated mice. To this end, the co-localization of CXCR3 with CXCL11 was analyzed using laser confocal microscopy. As shown in Fig. 5, clear evidence was provided that there was an interaction between CXCR3 and CXCL11 in UUO-induced damage. In sham mice, CXCR3- and CXCL11-positive cells were at low levels, indicating minimal interaction (Fig. 5). The CXCL11- and CXCR3-positive cells significantly increased in the UUO-vehicle group compared with sham-vehicle/sorafenib group. The number of cells co-stained for CXCL11 and CXCR3 decreased markedly in the UUO-sorafenib mice. These results indicated that sorafenib treatment reduces renal fibrosis by blocking the CXCR3/CXCL11 axis in the UUO model.

\section{Effects of sorafenib on macrophage and myofibroblast interaction}

The pathophysiological involvement of macrophages in UUO-induced renal fibrosis was elucidated by observation of co-localized CD68 and $\alpha$-SMA using confocal microscopy. The results of double-color immunofluorescence staining showed that in sham mice, double positive CD68 and $\alpha$-SMA cells were very weak, indicating that there was minimal interaction between macrophages and fibroblasts. CD68-positive macrophages were observed neighboring or directly adhered to the $\alpha$-SMA-positive myofibroblasts in UUOvehicle mice. These results showed that the CD68-positive macrophages stimulate $\alpha$-SMApositive myofibroblast activation in UUO-induced renal fibrosis.

\section{Discussion}

The data presented in this study demonstrates that sorafenib has fibro-suppressive effects against renal fibrosis induced by UUO in mice, and these effects may be caused, at least in part, by its inhibitory action against CXCL11/CXCR3, which mediates macrophage infiltration into kidneys, via suppression of the interactions between macrophage and myofibroblasts and the TGF $\beta 1$-mediated fibrogenic pathway.

UUO is an experimental model of renal injury that mimics the complex pathophysiology of chronic obstructive nephropathy [20]. It has been well established that UUO causes tubulointerstitial damage through many molecular mechanisms, including interstitial inflammatory macrophage infiltration, myofibroblast activation, and ECM deposition $[2,21]$. Monocyte chemotactic protein-1 (MCP-1) is known to play an important role in this process, leading to macrophage infiltration into the injured kidney and thereby accelerating fibrosis development [22]. Thus, we investigated the effect of sorafenib on the expression of MCP1 and IL-1 $\beta$, which are mainly derived from macrophages. However, these pro-inflammatory cytokines were predominantly derived from tubular epithelial cells, indicating these proinflammatory cytokines were not produced by macrophages. However, we found that the 


\section{Cellular Physiology Cell Physiol Biochem 2016;39:1837-1849 \\ and Biochemistry \begin{tabular}{l|l|l} 
DOI: 10.1159/000447883 & $\begin{array}{l}\text { C } 2016 \text { The Author(s). Published by S. Karger AG, Basel } \\
\text { www.karger.com/cpb }\end{array}$ \\
\hline
\end{tabular} \\ Ma et al.: Sorafenib and Macrophage Infiltration}

localization of CXCR3 was almost identical with that of CD68 from immunohistochemistry analyses, indicating that CXCR3 may mediate the macrophage infiltration.

Analysis of the expression of the CXCR3-binding ligands CXCL9, CXCL10, and CXCL11 revealed that these chemokines showed very low expression levels in the kidney (except for CXCL11). It was reported that CXCL11, but not CXCL9 or CXCL10, was up-regulated significantly in the bronchi alveolar and plasma in lung fibrosis. Furthermore, these findings were paralleled by a statistically significantly increase in lung gene expression of CXCL11 but not CXCL9 or CXCL10. These results suggest that CXCL11 plays an important role in fibrosis $[23,24]$. However, the effects of CXCL11 on the pathogenesis of renal fibrosis have not been elucidated. Our results showed that CXCL11 could interact with CXCR3 in UUO-induced renal fibrosis. Combined with these results, we hypothesized that the CXCL11/CXCR3 axis plays an important role in macrophage infiltration in renal fibrosis.

Progressive chronic kidney disease is characterized by inflammatory macrophage infiltration, myofibroblast activation, and fibrosis [21, 25, 26]. Macrophages could activate myofibroblasts by producing and releasing a variety of mediators, such as pro-inflammatory cytokines and reactive oxygen species, which fulfill a crucial function in the inflammatory responses of the kidneys [4]. However, few studies have examined the interactions between macrophages and myofibroblasts in vivo. To overcome these obstacles, we performed double immunofluorescence analyses to characterize the interaction between macrophages and myofibroblasts. Our results provided clear evidence of the interactions between macrophages and myofibroblasts.

Sorafenib has been shown to improve lung and liver fibrosis $[4,10,11,27]$. Sorafenib could reduce the rate of apoptosis and EMT in TGF- $\beta$-induced NRK-52E cells and ameliorate renal fibrosis in a UUO model [12]. We also observed $\alpha$-SMA-positive tubular epithelial cells (Fig. 6), indicating EMT in UUO-induced renal fibrosis. We provided evidence that sorafenib inhibited myofibroblast activation in the murine pathogenesis of renal fibrosis induced by UUO in vivo. TGF- $\beta 1$ is a multifunctional cytokine that plays a crucial role in myofibroblast activation in progression of renal fibrosis [4]. Activated TGF- $\beta 1$ binds to its type I and II receptors and induces activation of the downstream Smad-dependent pathway and myofibroblast activation [15, 28-31]. The myofibroblast activation coupled with excessive ECM production is perpetuated during fibrogenesis [32]. Considering the central role of activated myofibroblasts in renal fibrosis, we also evaluated the effect of sorafenib on TGF- $\beta 1$ signaling and collagen synthesis of fibroblasts. Here, we found that sorafenib could inhibit the TGF- $\beta 1$ signaling pathway, myofibroblast activation, and collagen production (Fig. 2), which is consistent with previous observations of the effects of sorafenib in various myofibroblasts. Based on these encouraging data generated from an animal model of renal fibrosis in this study, sorafenib may have considerable therapeutic benefits and may improve renal fibrosis. Taking into account the beneficial effects of sorafenib in experimental studies of hepatic cirrhosis and pulmonary hypertension, we believe this chemical may have a much broader role in clinical medicine.

In summary, we demonstrated that sorafenib ameliorates UUO-mediated renal fibrosis by inhibiting macrophage infiltration, suggesting an attractive pharmacological tool for the treatment of renal fibrosis.

\section{Acknowledgements}

This work was mainly supported in whole or part by the Putuo Hospital (no. 2014YJ001, to C. Liu), the "Budgeted Program" of Shanghai University of Traditional Chinese Medicine (no. 14YSN67, to C. Liu), the Shanghai Municipal Public Health Bureau (no. 201440370, to D. Xue), the Shanghai Putuo Science and Technology Commission Project (no. 2011PTKW006, to D. Xue), and the National Natural Science Foundacion of China (no. 81673788, to C. Liu). 


\section{Cellular Physiology Cell Physiol Biochem 2016;39:1837-1849 \begin{tabular}{ll|l} 
and Biochem 10.1159/000447883 & $\begin{array}{l}\text { @ 2016 The Author(s). Published by S. Karger AG, Basel } \\
\text { www.karger.com/cpb }\end{array}$ \\
\hline
\end{tabular} Ma et al.: Sorafenib and Macrophage Infiltration}

\section{Disclosure Statement}

The authors report no conflict of interest in this work.

\section{References}

1 Sakuraya K, Endo A, Someya T, Hirano D, Murano Y, Fujinaga S, Ohtomo Y, Shimizu T: The synergistic effect of mizoribine and a direct renin inhibitor, aliskiren, on unilateral ureteral obstruction induced renal fibrosis in rats. J Urol 2014;191:1139-1146.

2 Xiao H, Si L, Liu W, Li N, Meng G, Yang N, Chen X, Zhou Y, Shen H: The effects of adenosine A2A receptor knockout on renal interstitial fibrosis in a mouse model of unilateral ureteral obstruction. Acta Histochem 2013;115:315-319.

3 Kui Tan T, Zheng G, Hsu T, Ra Lee S, Zhang J, Zhao Y, Tian X, Wang Y, Min Wang Y, Cao Q Wang Y, Lee VW, Wang C, Zheng D, Alexander SI, Thompson E, Harris DCH: Matrix metalloproteinase-9 of tubular and macrophage origin contributes to the pathogenesis of renal fibrosis via macrophage recruitment through osteopontin cleavage. Lab Invest 2013;93:434-449.

4 Tan TK, Zheng G, Hsu T, Wang Y, Lee VWS, Tian X, Wang Y, Cao Q, Wang Y, Harris DCH: Macrophage matrix metalloproteinase-9 mediates Epithelial-Mesenchymal transition in vitro in murine renal tubular cells. Am J Pathol 2010;176:1256-1270.

5 Choi DE, Jeong JY, Lim BJ, Chang Y, Na K, Shin Y, Lee KW: Aliskiren ameliorates renal inflammation and fibrosis induced by unilateral ureteral obstruction in mice. J Urol 2011;186:694-701.

6 Schütte K, Zimmermann L, Bornschein J, Csepregi A, Rühl R, Ricke J, Malfertheiner P: Sorafenib therapy in patients with advanced hepatocellular carcinoma in advanced liver cirrhosis. Digestion 2011;83:275-282.

7 Pinter M, Sieghart W, Reisegger M, Wrba F, Peck-Radosavljevic M: Sorafenib in unresectable intrahepatic cholangiocellular carcinoma: A case report. Wien Klin Wochenschr 2011;123:61-64.

8 Hennenberg M, Trebicka J, Stark C, Kohistani AZ, Heller J, Sauerbruch T: Sorafenib targets dysregulated Rho kinase expression and portal hypertension in rats with secondary biliary cirrhosis. Br J Pharmacol 2009;157:258-270.

9 Hsu C, Shen Y, Lin Z, Chen P, Shao Y, Ding Y, Hsu C, Cheng A: Phase II study of combining sorafenib with metronomic tegafur/uracil for advanced hepatocellular carcinoma. J Hepatol 2010;53:126-131.

10 Hong F, Chou H, Fiel MI, Friedman SL: Antifibrotic activity of sorafenib in experimental hepatic fibrosis: Refinement of inhibitory targets, dosing, and window of efficacy in vivo. Dig Dis Sci 2012;58:257-264.

11 Wang Y, Gao J, Zhang D, Zhang J, Ma J, Jiang H: New insights into the antifibrotic effects of sorafenib on hepatic stellate cells and liver fibrosis. J Hepatol 2010;53:132-144.

12 Jia L, Ma X, Gui B, Ge H, Wang L, Ou Y, Tian L, Chen Z, Duan Z, Han J, Fu R: Sorafenib ameliorates renal fibrosis through inhibition of TGF- $\beta$-Induced Epithelial-Mesenchymal transition. PLos One 2015;10:e117757.

13 Liu Y, Wang Y, Zhuang F, Xian S, Fang J, Su W, Zhang W: Immunosuppression effects of bone marrow mesenchymal stem cells on renal interstitial injury in rats with unilateral ureteral obstruction. Cell Immunol 2012;276:144-152.

14 Ikezumi Y, Suzuki T, Hayafuji S, Okubo S, Nikolic-Paterson DJ, Kawachi H, Shimizu F, Uchiyama M: The sialoadhesin (CD169) expressing a macrophage subset in human proliferative glomerulonephritis. Nephrol Dial Transplant 2005;20:2704-2713.

15 Sung WJ, Kim K, Kim Y, Chang Y, Lee IH, Park K: Antifibrotic effect of synthetic Smad/Sp1 chimeric decoy oligodeoxynucleotide through the regulation of epithelial mesenchymal transition in unilateral ureteral obstruction model of mice. Exp Mol Pathol 2013;95:136-143.

16 Nishida M, Hamaoka K: Macrophage phenotype and renal fibrosis in obstructive nephropathy. Nephron Exp Nephrol 2008;110:e31-e36.

17 Zimmerman DL, Zimpelmann J, Xiao F, Gutsol A, Touyz R, Burns KD: The effect of angiotensin-(1-7) in mouse unilateral ureteral obstruction. Am J Pathol 2015;185:729-740.

18 Hatano M, Sasaki S, Ohata S, Shiratsuchi Y, Yamazaki T, Nagata K, Kobayashi Y: Effects of Kupffer celldepletion on Concanavalin A-induced hepatitis. Cell Immunol 2008;251:25-30. 


\section{Cellular Physiology Cell Physiol Biochem 2016;39:1837-1849 \begin{tabular}{ll|l} 
and Biochemistry $10.1159 / 000447883$ & $\begin{array}{l}\text { @ 2016 The Author(s). Published by S. Karger AG, Basel } \\
\text { www.karger.com/cpb }\end{array}$ \\
\hline
\end{tabular} \\ Ma et al.: Sorafenib and Macrophage Infiltration}

19 Torraca V, Cui C, Boland R, Bebelman JP, van der Sar AM, Smit MJ, Siderius M, Spaink HP, Meijer AH: The CXCR3/CXCL11 signaling axis mediates macrophage recruitment and dissemination of mycobacterial infection. Dis Model Mech 2015;8:253-269

20 Tsutsumi T, Adachi M, Nikawadori M, Morishige J, Tokumura A: Presence of bioactive lysophosphatidic acid in renal effluent of rats with unilateral ureteral obstruction. Life Sci 2011;89:195-203.

21 Uchida Y, Miyajima A, Kikuchi E, Kozakai N, Kosaka T, Ieda M, Fukuda K, Ohigashi T, Oya M: Renal damage inhibited in mice lacking angiotensinogen gene subjected to unilateral ureteral obstruction. Urology 2009;74:938-943.

22 Tieu BC, Lee C, Sun H, LeJeune W, Recinos A, Ju X, Spratt H, Guo D, Milewicz D, Tilton RG, Brasier AR: An adventitial IL-6/MCP1 amplification loop accelerates macrophage-mediated vascular inflammation leading to aortic dissection in mice. J Clin Invest 2009;119:3637-3651.

23 Burdick MD, Murray LA, Keane MP, Xue YY, Zisman DA, Belperio JA, Strieter RM: CXCL11 attenuates bleomycin-induced pulmonary fibrosis via inhibition of vascular remodeling. Am J Resp Crit Care 2005;171:261-268.

24 Chen Y, Huang Y, Reiberger T, Duyverman AM, Huang P, Samuel R, Hiddingh L, Roberge S, Koppel C, Lauwers GY, Zhu AX, Jain RK, Duda DG: Differential effects of sorafenib on liver versus tumor fibrosis mediated by stromal-derived factor 1 alpha/C-X-C receptor type 4 axis and myeloid differentiation antigen-positive myeloid cell infiltration in mice. Hepatology 2014;59:1435-1447.

25 Zeng J, Dou Y, Guo J, Wu X, Dai Y: Paeoniflorin of Paeonia lactiflora prevents renal interstitial fibrosis induced by unilateral ureteral obstruction in mice. Phytomedicine 2013;20:753-759.

26 Ito K, Yoshii H, Asano T, Seta K, Mizuguchi Y, Yamanaka M, Tokonabe S, Hayakawa M, Asano T: Adrenomedullin increases renal nitric oxide production and ameliorates renal injury in mice with unilateral ureteral obstruction. J Urol 2010;183:1630-1635.

27 Chen Y, Zhang X, Bai J, Gai L, Ye X, Zhang L, Xu Q, Zhang Y, Xu L, Li H, Ding X: Sorafenib ameliorates bleomycin-induced pulmonary fibrosis: Potential roles in the inhibition of epithelial-mesenchymal transition and fibroblast activation. Cell Death and Dis 2013;4:e665. doi: 10.1038/cddis.2013.154

28 Ali BH, Al Za"abi M, Adham SA, Yasin J, Nemmar A, Schupp N: Therapeutic effect of chrysin on AdenineInduced chronic kidney disease in rats. Cell Physiol Biochem 2016;38:248-257.

29 Wang D, Guan M, Zheng Z, Li W, Lyv F, Pang R, Xue Y: Transcription factor egr1 is involved in high GlucoseInduced proliferation and fibrosis in rat glomerular mesangial cells. Cell Physiol Biochem 2016;36:20932107.

30 Feger M, Alesutan I, Castor T, Mia S, Musculus K, Voelkl J, Lang F: Inhibitory Effect of NH4Cl Treatment on Renal Tgf $\beta 1$ Signaling Following Unilateral Ureteral Obstruction. Cell Physiol Biochem 2015;37:955-964.

31 Garc A-S Nchez O, Sancho-Mart Nez SM, L Pez-Novoa JM, L Pez-Hern Ndez FJ: Activation of the ALK-5 Pathway is not per se Sufficient for the Antiproliferative Effect of TGF- $\beta 1$ on Renal Tubule Epithelial Cells. Cell Physiol Biochem 2015;37:1231-1239.

32 Su T, Shiau C, Jao P, Liu C, Liu C, Tai W, Jeng Y, Yang H, Tseng T, Huang H, Cheng H, Chen P, Chen K, Kao J, Chen D: Sorafenib and its derivative SC-1 exhibit antifibrotic effects through signal transducer and activator of transcription 3 inhibition. Proc Natl Acad Sci U S A 2015;112:7243-7248. 